continuity) are specially marked, while there are appendixes giving more advanced mathematical proofs for those able to follow them. In my opinion, this system has worked well in Vol. 3. It is a sign of the times that electrical engineers and technicians are now expected to have some knowledge of the structure of matter. The rapidity with which new materials and methods are being produced makes the acquisition of this knowledge very desirable. In this book, a very readable and accurate (to the best of my rather limited knowledge) introduction to this subject is given.

The author is, of course, an expert on valves and acquainted with recent developments. The chapters on valves are a happy blend of theory and practice, being illustrated by references to modern types, and numerical values are stressed throughout. A sug. gested explanation is given for the fact that oxidecoated cathodes can be used at high voltages and currents under pulsed conditions. After discussing high-frequency effects in 'conventional' valves, klystrons and magnetrons are described. Noise is given a fuller treatment than is usual in a general book of this kind. Space does not permit of more than a brief description of gas-filled devices.

This is one of the first general text-books to contain a treatment of transistors. The difficulties which beset an author writing on a subject like electronics can be seen from p. 199, where it is stated that the problems of quantity production of the germanium triode have not yet been solved; and on p. 216 it is suggested that point-contact transistors are already in use experimentally, but the junction transistor "if it can be made in quantity, will have many important uses". I agree that there is much to be done to make cheaper and more consistent transistors in large quantities; but the above statements scarcely represent the present position. They can be understood, however, when it is observed that, although the book was published in 1955, the author's preface is dated April 1952. Interesting chapters on electron optics, cathode-ray tubes and photoelectric devices complete the book. The rationalized M.K.s. system of units is used and standard symbols, except that $x$ is still retained for permittivity. Needless to say, there are no 'jars'!

The book can be thoroughly recommended for any of the three classes of reader for whom it is intended. The style of writing is easy, the author seeming to be in close touch with the reader. The diagrams are clear and fulfil their purpose.

C. R. STONER

\section{OPTICAL INVESTIGATION OF DIAMOND SURFACES}

The Microstructures of Diamond Surfaces

By Prof. S. Tolansky. Pp. viii $+67+143$ plates. (London: N.A.G. Press, Ltd., 1955.) 40s. net.

NVESTIGATIONS of the surface topography of crystals have yielded many results of fundamental significance in recent years. The observations of growth spirals on the surfaces of crystals of beryl and carborundum which were made in Prof. S. Tolansky's laboratory provided some of the first evidence to support Frank's theory of the role of screw dislocations in crystal growth. Since then, it has been realized to an ever-increasing extent that the study of the microtopography of the surfaces which result from the final stages of crystal growth and from subsequent controlled sublimation, thermal etching, chemical attack or progressive dissolution provides one of the most powerful means of deriving information about the distribution of dislocations and their behaviour in crystals.

By his development and application of a series of essentially simple--yet surprisingly powerful-optical techniques which permit the quantitative study of surface features from the coarsest down almost to molecular dimensions, Prof. Tolansky has made a valuable contribution to this aspect of the physics and chemistry of solids. He has taken advantage of the opportunity provided by the publication of this volume to demonstrate the application of his methods to the study of the structures of natural surfaces, of cleavage surfaces, and of etched, polished and indented surfaces of diamond crystals. Multiplebeam interferometry, the powerful crossed-fringes technique and the light-profile method are clearly and briefly discussed and their utility and limitations outlined. The application of each technique to a number of different problems is well illustrated, and one of the most impressive features of the volume is the inclusion of one hundred and forty-three photomicrographs of outstanding quality.

The distribution of trigons on natural surfaces of diamond and the microtopography of these features as determined by the different optical methods provides one of the main topics of the book. The author expresses the very definite opinion that the trigons have their origin in phenomena which occur during the final stages of crystal growth, and that they are not to be attributed to localized attack on the surface as a result of changing physico-chemical conditions in the medium surrounding the crystals after the conclusion of crystal growth. $\mathrm{He}$ illustrates the experiments on controlled oxidation with molten potassium nitrate which have been carried out in his own laboratory with a further series of fine photomicrographs. No evidence has been found for the participation of screw dislocations in the processes of crystal growth and controlled oxidation in diamond.

The cleavage of diamond is of considerable interest to the physicist, the mineralogist and the lapidary, and the observation that Type 1 diamonds which absorb below $3000 \mathrm{~A}$. have an imperfect cleavage compared with Type 2 diamonds, which only begin to absorb below $2000 \mathrm{~A}$., is noteworthy. It is suggested that this may be correlated with the supposed greater purity of the Type 2 diamonds. In other substances, there is also often a correlation between the perfection of cleavage surfaces and the purity of the specimens.

The chapters on the formation of ring cracks on diamond surfaces by the application of a relatively lightly loaded hemispherical diamond indenter and on the comparative properties of the different crystallographic surfaces of diamond and the state of polish which can be achieved on them, although representing only an interim report on work which is still in progress, will interest engineers and metallurgists who are concerned with industrial diamond tools and indenters.

With his adequate elementary introduction to the optical techniques, and his beautiful illustrations of their application, the author can scarcely fail in his main objective in writing this book, which was to interest as wide a variety of workers as possible in the methods for the investigation of the microtopography of surfaces which have been developed in his laboratory.
J. W. Mitchell 